\title{
Modified Tseng's Extragradient Methods for Solving Pseudo-Monotone Variational Inequalities
}

\author{
Duong Viet Thong* $\quad$ Phan Tu Vuong ${ }^{\dagger}$
}

February 12, 2019

\begin{abstract}
We propose two modified Tseng's extragradient methods (also known as ForwardBackward-Forward methods) for solving non-Lipschitzian and pseudo-monotone variational inequalities in real Hilbert spaces. Under mild and standard conditions, we obtain the weak and strong convergence of the proposed methods. Numerical examples for illustrating the behavior of the proposed methods are also presented.
\end{abstract}

Keywords: Forward-Backward-Forward method, Extragradient method, Mann type method, Variational inequality, Pseudomonotone operator.

\section{Introduction}

In this paper, we are interested in the classical variational inequality (VI) [11, 12], which consists in finding a point $x^{*} \in C$ such that

$$
\left\langle A x^{*}, x-x^{*}\right\rangle \geq 0 \forall x \in C,
$$

where $C$ is a nonempty closed convex subset in a real Hilbert space $H$, and $A: H \rightarrow H$ is a single-valued mapping. We denote (1) by $\operatorname{VI}(C, A)$ and its the solution set by $\Omega$, which is assumed to be non-empty.

Variational inequalities are fundamental in a broad range of mathematical and applied sciences; the theoretical and algorithmic foundations as well as the applications of variational inequalities have been extensively studied in the literature and continue to attract intensive research $[10,18,19]$. For the current state of the art in finite dimensional setting, see for instance [10] and the extensive list of references therein.

Solution methods solving the variational inequality (1) have been developed extensively in the literature $[4,5,6,10,16,17,18,19,23,24,28,30,31,33,34]$. The simplest method is the classical projection algorithm, which generates an iterative sequence via

$$
x_{n+1}=P_{C}\left(x_{n}-\lambda A x_{n}\right), \quad \forall n \geq 0,
$$

*Applied Analysis Research Group, Faculty of Mathematics and Statistics, Ton Duc Thang University, Ho Chi Minh City, Vietnam. Email: duongvietthong@tdtu.edu.vn

${ }^{\dagger}$ Faculty of Mathematics, University of Vienna, Oskar-Morgenstern-Platz 1, 1090 Vienna, Austria. Email: vuong.phan@univie.ac.at 
where $P_{C}$ denotes the metric projection from $H$ onto $C$. This method is an extension of the projected gradient method for solving optimization problems. It is known that the convergence of this projection method only holds under quite restrictive assumptions that $A$ is $L$-Lipschitz continuous and $\alpha$-strongly (pseudo)-monotone and the setpsize $\lambda$ is chosen satisfying $\lambda \in\left(0, \frac{2 \alpha}{L^{2}}\right)[10,16]$.

Korpelevich [20] (and also independently Antipin [1]) proposed a double projection method in Euclidean space, known as the extragradient method for solving VIs when $A$ is monotone and $L$-Lipschitz continuous

$$
\left\{\begin{array}{l}
x_{0} \in C, \\
y_{n}=P_{C}\left(x_{n}-\lambda A x_{n}\right), \\
x_{n+1}=P_{C}\left(x_{n}-\lambda A y_{n}\right),
\end{array} \quad \forall n \geq 0,\right.
$$

where $\lambda \in\left(0, \frac{1}{L}\right)$. It is well known that the extragradient method can be applied to solve pseudo-monotone, Lipschitz continuous VIs in finite dimensional spaces [10, 28]. The weak convergence of this method in infinite dimensional Hilbert spaces was studied in [7] under an additional assumption that $A$ is sequentially weak-strong continuous, i.e., $A$ maps a weakly convergent sequence to a strongly convergent sequence. This assumption is rather strong and is not satisfied even for a simple example when $A$ is the identity operator. In [33], the author has weakened this assumption to sequentially weak-weak continuity of $A$.

The extragradient method and its variants require (at least) two projections per iteration. Censor, Gibali and Reich [4, 5, 6] proposed the following scheme, called subgradient extragradient method

$$
\left\{\begin{array}{rl}
y_{n} & =P_{C}\left(x_{n}-\lambda F\left(x_{n}\right)\right), \\
x_{n+1} & =P_{T_{n}}\left(x_{n}-\lambda F\left(y_{n}\right)\right)
\end{array} \quad \forall n \geq 0,\right.
$$

where

$$
T_{n}=\left\{w \in H,\left\langle x_{n}-\lambda F\left(x_{n}\right)-y_{n}, w-y_{n}\right\rangle \leq 0\right\} .
$$

Since the projection onto the half-space $T_{n}$ can be explicitly calculated [2], the subgradient extragradient requires only one projection per iteration. This method converges for pseudo-monotone VIs in finite dimensional Euclidean spaces [6] and monotone VIs in infinite dimensional Hilbert spaces $[4,5]$.

An alternative method of the extragradient method is the following remarkable scheme studied by Tseng [32], which also requires only one projection per iteration

$$
\left\{\begin{array}{rl}
y_{n} & =P_{C}\left(x_{n}-\lambda F\left(x_{n}\right)\right), \\
x_{n+1} & =y_{n}+\lambda\left(F\left(x_{n}\right)-F\left(y_{n}\right)\right)
\end{array} \quad \forall n \geq 0 .\right.
$$

The weak convergence of Tseng's extragradient method (also known as the ForwardBackward-Forward method) for solving monotone Lipschitz continuous VIs was established in [32], and is recently studied in [3] for solving pseudo-monotone Lipschitz continuous VIs under sequentially weak-weak continuity of $A$. 
The aim of this paper has two folds. We first incorporate the Tseng's extragradient method studied in $[3,32]$ with a suitable linesearch to remove the dependence on the Lipschitz continuity modulus of $A$ when choosing stepsize $\lambda$. We also weaken the Lipschitz continuity of $A$ to the uniform continuity. This is crucial when the operator is not Lipschitz continuous and/or the Lipschitz modulus is difficult to estimate in advance. Doing so, we obtain the weak convergence of the iterative sequence. As we are working in infinite dimensional Hilbert spaces, the strong convergence is essential. Therefore, in the second part of the paper, we combine the linesearch method with a Mann-type iteration step to obtain the strong convergence of the iterative sequence.

The rest of the paper is organized as follows. We first recall some basic definitions and results in Section 2. The weak convergence method and its convergence analysis are presented in Section 3. Section 4 contains the analysis of the strong convergence method. In Section 5 we present some elementary numerical experiments which demonstrate the performances of the proposed methods. Finally, we give some conclusion remarks in the last section.

\section{Preliminaries}

Let $H$ be a real Hilbert space and $C$ be a nonempty, closed and convex subset of $H$. The weak convergence of $\left\{x_{n}\right\}_{n=1}^{\infty}$ to $x$ is denoted by $x_{n} \rightarrow x$ as $n \rightarrow \infty$, while the strong convergence of $\left\{x_{n}\right\}_{n=1}^{\infty}$ to $x$ is written as $x_{n} \rightarrow x$ as $n \rightarrow \infty$. For each $x, y, z \in H$ and for all $\alpha, \beta, \gamma \in[0,1]$ with $\alpha+\beta+\gamma=1$, we have

$$
\begin{gathered}
\|x+y\|^{2} \leq\|x\|^{2}+2\langle y, x+y\rangle \\
\|\alpha x+(1-\alpha) y\|^{2}=\alpha\|x\|^{2}+(1-\alpha)\|y\|^{2}-\alpha(1-\alpha)\|x-y\|^{2} \\
\|\alpha x+\beta y+\gamma z\|^{2}=\alpha\|x\|^{2}+\beta\|y\|^{2}+\gamma\|z\|^{2}-\alpha \beta\|x-y\|^{2}-\alpha \gamma\|x-z\|^{2}-\beta \gamma\|y-z\|^{2} .
\end{gathered}
$$

Definition 1. Let $T: H \rightarrow H$ be an operator.

1. The operator $T$ is called L-Lipschitz continuous with $L>0$ if

$$
\|T x-T y\| \leq L\|x-y\| \quad \forall x, y \in H
$$

if $L=1$ then the operator $T$ is called nonexpansive and if $L \in(0,1), T$ is called contraction.

2. The operator $T$ is called monotone if

$$
\langle T x-T y, x-y\rangle \geq 0 \quad \forall x, y \in H
$$

3. The operator $T$ is called pseudo-monotone if

$$
\langle T x, y-x\rangle \geq 0 \Longrightarrow\langle T y, y-x\rangle \geq 0 \quad \forall x, y \in H
$$

4. The operator $T$ is called sequentially weakly continuous if for each sequence $\left\{x_{n}\right\}$ we have: $\left\{x_{n}\right\}$ converges weakly to $x$ implies T $x_{n}$ converges weakly to $T x$. 

that

For every point $x \in H$, there exists a unique nearest point in $C$, denoted by $P_{C} x$ such

$$
\left\|x-P_{C} x\right\| \leq\|x-y\| \forall y \in C .
$$

$P_{C}$ is called the metric projection of $H$ onto $C$. It is well known that $P_{C}$ is nonexpansive and the following properties hold.

Lemma 1. ([13]) Let $C$ be a nonempty closed convex subset of a real Hilbert space $H$. Given $x \in H$ and $z \in C$. Then $z=P_{C} x \Longleftrightarrow\langle x-z, z-y\rangle \geq 0 \forall y \in C$.

Lemma 2. ([13]) Let $C$ be a closed and convex subset in a real Hilbert space $H, x \in H$. Then

i) $\left\|P_{C} x-P_{C} y\right\|^{2} \leq\left\langle P_{C} x-P_{C} y, x-y\right\rangle \forall y \in C$;

ii) $\left\|P_{C} x-y\right\|^{2} \leq\|x-y\|^{2}-\left\|x-P_{C} x\right\|^{2} \forall y \in C$;

iii) $\left\langle\left(I-P_{C}\right) x-\left(I-P_{C}\right) y, x-y\right\rangle \geq\left\|\left(I-P_{C}\right) x-\left(I-P_{C}\right) y\right\|^{2} \forall y \in C$.

The following Lemmas are useful for the convergence of our proposed methods.

Lemma 3. ([9]) For $x \in H$ and $\alpha \geq \beta>0$ the following inequalities hold.

$$
\begin{aligned}
& \frac{\left\|x-P_{C}(x-\alpha A x)\right\|}{\alpha} \leq \frac{\left\|x-P_{C}(x-\beta A x)\right\|}{\beta}, \\
& \left\|x-P_{C}(x-\beta A x)\right\| \leq\left\|x-P_{C}(x-\alpha A x)\right\| .
\end{aligned}
$$

Lemma 4. ([14, 15]) Let $H_{1}$ and $H_{2}$ be two real Hilbert spaces. Suppose $A: H_{1} \rightarrow H_{2}$ is uniformly continuous on bounded subsets of $H_{1}$ and $M$ is a bounded subset of $H_{1}$. Then $A(M)$ (the image of $M$ under $A$ ) is bounded.

Lemma 5. [8, Lemma 2.1] Consider the problem $V I(C, A)$ with $C$ being a nonempty, closed, convex subset of a real Hilbert space $H$ and $A: C \rightarrow H$ being pseudo-monotone and continuous. Then, $x^{*}$ is a solution of $\operatorname{VI}(C, A)$ if and only if

$$
\left\langle A x, x-x^{*}\right\rangle \geq 0 \quad \forall x \in C .
$$

Lemma 6. ([26]) Let $C$ be a nonempty set of $H$ and $\left\{x_{n}\right\}$ be a squence in $H$ such that the following two conditions hold:

i) for every $x \in C, \lim _{n \rightarrow \infty}\left\|x_{n}-x\right\|$ exists;

ii) every sequential weak cluster point of $\left\{x_{n}\right\}$ is in $C$.

Then $\left\{x_{n}\right\}$ converges weakly to a point in $C$.

The next technical lemma is very useful and used by many authors, for example Liu [21] and $\mathrm{Xu}$ [36]. Furthermore, a variant of Lemma 7 has already been used by Reich in [27].

Lemma 7. Let $\left\{a_{n}\right\}$ be sequence of nonnegative real numbers such that:

$$
a_{n+1} \leq\left(1-\alpha_{n}\right) a_{n}+\alpha_{n} b_{n},
$$

where $\left\{\alpha_{n}\right\} \subset(0,1)$ and $\left\{b_{n}\right\}$ is a sequence such that

a) $\sum_{n=0}^{\infty} \alpha_{n}=\infty$;

b) $\lim \sup _{n \rightarrow \infty} b_{n} \leq 0$.

Then $\lim _{n \rightarrow \infty} a_{n}=0$. 


\section{Weak Convergence Method}

Through this section, we make the following conditions on $\operatorname{VI}(C, A)$ :

Condition 1. The feasible set $C$ of $V I(C, A)$ is a nonempty, closed, and convex subset of the real Hilbert space $H$.

Condition 2. The operator $A: H \rightarrow H$ is a pseudo-monotone, sequentially weakly continuous on $C$, and uniformly continuous on bounded subsets of $H$.

Condition 3. The solution set of $V I(C, A)$ is nonempty, that is $\Omega \neq \emptyset$.

We are now in the position to present our first method.

\section{Algorithm 1.}

Initialization: Given $\gamma>0, l \in(0,1), \mu \in(0,1)$. Let $x_{1} \in C$ be arbitrary

Iterative Steps: Given the current iterate $x_{n}$, calculate $x_{n+1}$ as follows:

Step 1. Compute

$$
y_{n}=P_{C}\left(x_{n}-\lambda_{n} A x_{n}\right)
$$

where $\lambda_{n}:=\gamma l^{m_{n}}$ and $m_{n}$ is the smallest non-negative integer $m$ satisfying

$$
\gamma l^{m}\left\|A x_{n}-A y_{n}\right\| \leq \mu\left\|x_{n}-y_{n}\right\| .
$$

If $x_{n}=y_{n}$ or $A y_{n}=0$ then Stop, $y_{n}$ is a solution of $\operatorname{VI}(C, A)$. Otherwise

Step 2. Compute

$$
x_{n+1}=y_{n}-\lambda_{n}\left(A y_{n}-A x_{n}\right) .
$$

Set $n:=n+1$ and go to Step 1 .

We start the convergence analysis by proving that (9) terminates after finite steps.

Lemma 8. Assume that Conditions 1-3 hold. Then the Armijo-line search rule (9) is well defined. In addition, we have $\lambda_{n} \leq \gamma$.

Proof. If $x_{n} \in \Omega$ then $x_{n}=P_{C}\left(x_{n}-\gamma A x_{n}\right)$, therefore (9) holds with $m=0$. If $x_{n} \notin \Omega$ and assume the contrary that for all $m$ we have

$$
\gamma l^{m}\left\|A x_{n}-A P_{C}\left(x_{n}-\gamma l^{m} A x_{n}\right)\right\|>\mu\left\|x_{n}-P_{C}\left(x_{n}-\gamma l^{m} A x_{n}\right)\right\| .
$$

This implies that

$$
\left\|A x_{n}-A P_{C}\left(x_{n}-\gamma l^{m} A x_{n}\right)\right\|>\mu \frac{\left\|x_{n}-P_{C}\left(x_{n}-\gamma l^{m} A x_{n}\right)\right\|}{\gamma l^{m}} .
$$

We consider two possibilities of $x_{n}$. First, if $x_{n} \in C$, then since $P_{C}$ ans $A$ are continuous, we have $\lim _{m \rightarrow \infty}\left\|x_{n}-P_{C}\left(x_{n}-\gamma l^{m} A x_{n}\right)\right\|=0$. From the uniform continuity of the operator $A$ on bounded subsets of $H$ it implies that

$$
\lim _{m \rightarrow \infty}\left\|A x_{n}-A P_{C}\left(x_{n}-\gamma l^{m} A x_{n}\right)\right\|=0 .
$$


Combining (11) and (12) we get

$$
\lim _{m \rightarrow \infty} \frac{\left\|x_{n}-P_{C}\left(x_{n}-\gamma l^{m} A x_{n}\right)\right\|}{\gamma l^{m}}=0 .
$$

Setting $z_{m}=P_{C}\left(x_{n}-\gamma l^{m} A x_{n}\right)$ we have

$$
\left\langle z_{m}-x_{n}+\gamma l^{m} A x_{n}, x-z_{m}\right\rangle \geq 0 \quad \forall x \in C .
$$

This implies that

$$
\left\langle\frac{z_{m}-x_{n}}{\gamma l^{m}}, x-z_{m}\right\rangle+\left\langle A x_{n}, x-z_{m}\right\rangle \geq 0 \quad \forall x \in C .
$$

Taking the limit $m \rightarrow \infty$ in (14) and using (13) we obtain

$$
\left\langle A x_{n}, x-x_{n}\right\rangle \geq 0 \forall x \in C,
$$

which implies that $x_{n} \in \Omega$. This is a contradiction. Now, if $x_{n} \notin C$, then we have

$$
\lim _{m \rightarrow \infty}\left\|x_{n}-P_{C}\left(x_{n}-\gamma l^{m} A x_{n}\right)\right\|=\left\|x_{n}-P_{C} x_{n}\right\|>0 .
$$

and

$$
\lim _{m \rightarrow \infty} \gamma l^{m}\left\|A x_{n}-A P_{C}\left(x_{n}-\gamma l^{m} A x_{n}\right)\right\|=0 .
$$

Combining (10), (15) and (16) we get a contradiction.

Remark 1. 1. In the proof of Lemma 8 we do not need the pseudo-monotonicity of $A$.

2. Note that if $x_{n}=y_{n}$ then $x_{n}$ is a solution of $V I(C, A)$. Indeed, we have $0<\lambda_{n} \leq \gamma$, which together with Lemma 3 we get

$$
0=\frac{\left\|x_{n}-y_{n}\right\|}{\lambda_{n}}=\frac{\left\|x_{n}-P_{C}\left(x_{n}-\lambda_{n} A x_{n}\right)\right\|}{\lambda_{n}} \geq \frac{\left\|x_{n}-P_{C}\left(x_{n}-\gamma A x_{n}\right)\right\|}{\gamma} .
$$

This implies that $x_{n}$ is a solution of $\operatorname{VI}(C, A)$.

The following Lemma states that the sequence $\left\{x_{n}\right\}$ is Fejér monotone with respect to the solution set $\Omega$.

Lemma 9. Let $\left\{x_{n}\right\}$ be a sequence generated by Algorithm 1. Then for every $p \in \Omega$ it holds

$$
\left\|x_{n+1}-p\right\|^{2} \leq\left\|x_{n}-p\right\|^{2}-\left(1-\mu^{2}\right)\left\|x_{n}-y_{n}\right\|^{2} .
$$

Proof. We have

$$
\begin{gathered}
\left\|x_{n+1}-p\right\|^{2}=\left\|y_{n}-\lambda_{n}\left(A y_{n}-A x_{n}\right)-p\right\|^{2} \\
=\left\|y_{n}-p\right\|^{2}+\lambda \lambda_{n}^{2}\left\|A y_{n}-A x_{n}\right\|^{2}-2 \lambda_{n}\left\langle y_{n}-p, A y_{n}-A x_{n}\right\rangle \\
=\left\|x_{n}-p\right\|^{2}+\left\|x_{n}-y_{n}\right\|^{2}+2\left\langle y_{n}-x_{n}, x_{n}-p\right\rangle \\
\quad+\lambda{ }_{n}^{2}\left\|A y_{n}-A x_{n}\right\|^{2}-2 \lambda_{n}\left\langle y_{n}-p, A y_{n}-A x_{n}\right\rangle \\
=\left\|x_{n}-p\right\|^{2}+\left\|x_{n}-y_{n}\right\|^{2}-2\left\langle y_{n}-x_{n}, y_{n}-x_{n}\right\rangle+2\left\langle y_{n}-x_{n}, y_{n}-p\right\rangle \\
\quad+\lambda_{n}^{2}\left\|A y_{n}-A x_{n}\right\|^{2}-2 \lambda_{n}\left\langle y_{n}-p, A y_{n}-A x_{n}\right\rangle \\
=\left\|x_{n}-p\right\|^{2}-\left\|x_{n}-y_{n}\right\|^{2}+2\left\langle y_{n}-x_{n}, y_{n}-p\right\rangle \\
\quad+\lambda_{n}^{2}\left\|A y_{n}-A x_{n}\right\|^{2}-2 \lambda_{n}\left\langle y_{n}-p, A y_{n}-A x_{n}\right\rangle .
\end{gathered}
$$


Since $y_{n}=P_{C}\left(x_{n}-\lambda_{n} A x_{n}\right)$ we obtain

$$
\left\langle y_{n}-x_{n}+\lambda_{n} A x_{n}, y_{n}-p\right\rangle \leq 0,
$$

or equivalently

$$
\left\langle y_{n}-x_{n}, y_{n}-p\right\rangle \leq-\lambda_{n}\left\langle A x_{n}, y_{n}-p\right\rangle \text {. }
$$

From (18) and (19), we get

$$
\begin{aligned}
\left\|x_{n+1}-p\right\|^{2} \leq & \left\|x_{n}-p\right\|^{2}-\left\|x_{n}-y_{n}\right\|^{2}-2 \lambda_{n}\left\langle A x_{n}, y_{n}-p\right\rangle+\lambda_{n}^{2}\left\|A y_{n}-A x_{n}\right\|^{2} \\
& -2 \lambda_{n}\left\langle y_{n}-p, A y_{n}-A x_{n}\right\rangle \\
= & \left\|x_{n}-p\right\|^{2}-\left\|x_{n}-y_{n}\right\|^{2}+\lambda_{n}^{2}\left\|A y_{n}-A x_{n}\right\|^{2}-2 \lambda_{n}\left\langle y_{n}-p, A y_{n}\right\rangle \\
\leq & \left\|x_{n}-p\right\|^{2}-\left\|x_{n}-y_{n}\right\|^{2}+\mu^{2}\left\|x_{n}-y_{n}\right\|^{2}-2 \lambda_{n}\left\langle y_{n}-p, A y_{n}\right\rangle \\
\leq & \left\|x_{n}-p\right\|^{2}-\left(1-\mu^{2}\right)\left\|x_{n}-y_{n}\right\|^{2}-2 \lambda_{n}\left\langle y_{n}-p, A y_{n}\right\rangle .
\end{aligned}
$$

Since $p \in \Omega$ we have $\left\langle A p, y_{n}-p\right\rangle \geq 0$, from the pseudo-monotonicity of $A$ we find

$$
\left\langle A y_{n}, y_{n}-p\right\rangle \geq 0 \text {. }
$$

Combining (20) and (21) we obtain

$$
\left\|x_{n+1}-p\right\|^{2} \leq\left\|x_{n}-p\right\|^{2}-\left(1-\mu^{2}\right)\left\|x_{n}-y_{n}\right\|^{2} .
$$

From Lemma 9, we have that for every $p \in \Omega, \lim _{n \rightarrow \infty}\left\|x_{n}-p\right\|$ exists. To obtain the weak convergence, following Lemma 6 , it remains to prove that every weak limit point of $\left\{x_{n}\right\}$ belongs to $\Omega$.

Lemma 10. Every weak limit point of $\left\{x_{n}\right\}$ is a solution of $V I(C, A)$.

Proof. Let $z$ be a weak limit point of $\left\{x_{n}\right\}$ and let $\left\{x_{n_{k}}\right\}$ be a subsequence of $\left\{x_{n}\right\}$ converges weakly to $z$. From Lemma 9 we have that $\left\{x_{n}\right\}$ is bounded and

$$
\lim _{n \rightarrow \infty}\left\|x_{n}-y_{n}\right\|=0 .
$$

Therefore, $y_{n_{k}} \rightarrow z$. Since $y_{n_{k}} \in C$ for all $k$ and $C$ is (weakly) closed we have $z \in C$. Since $y_{n_{k}}=P_{C}\left(x_{n_{k}}-\lambda_{n_{k}} A x_{n_{k}}\right)$ it holds

$$
\left\langle x_{n_{k}}-\lambda_{n_{k}} A x_{n_{k}}-y_{n_{k}}, x-y_{n_{k}}\right\rangle \leq 0 \quad \forall x \in C .
$$

or equivalently

$$
\frac{1}{\lambda_{n_{k}}}\left\langle x_{n_{k}}-y_{n_{k}}, x-y_{n_{k}}\right\rangle \leq\left\langle A x_{n_{k}}, x-y_{n_{k}}\right\rangle \forall x \in C .
$$

This implies that

$$
\frac{1}{\lambda_{n_{k}}}\left\langle x_{n_{k}}-y_{n_{k}}, x-y_{n_{k}}\right\rangle+\left\langle A x_{n_{k}}, y_{n_{k}}-x_{n_{k}}\right\rangle \leq\left\langle A x_{n_{k}}, x-x_{n_{k}}\right\rangle \forall x \in C .
$$

Now, we show that

$$
\liminf _{k \rightarrow \infty}\left\langle A x_{n_{k}}, x-x_{n_{k}}\right\rangle \geq 0
$$


Indeed, let us consider two possible cases. Suppose first that $\liminf _{k \rightarrow \infty} \lambda_{n_{k}}>0$. We have $\left\{x_{n_{k}}\right\}$ is a bounded sequence, $A$ is uniformly continuous on bounded subsets of $H$. By Lemma 5, we get that $\left\{A x_{n_{k}}\right\}$ is bounded. Taking $k \rightarrow \infty$ in (22) since $\left\|x_{n_{k}}-y_{n_{k}}\right\| \rightarrow 0$, we get

$$
\liminf _{k \rightarrow \infty}\left\langle A x_{n_{k}}, x-x_{n_{k}}\right\rangle \geq 0 .
$$

Assume now that liminf ${ }_{k \rightarrow \infty} \lambda_{n_{k}}=0$. Setting $z_{n_{k}}=P_{C}\left(x_{n_{k}}-\lambda_{n_{k}} \cdot l^{-1} A x_{n_{k}}\right)$, we have $\lambda_{n_{k}} l^{-1}>$ $\lambda_{n_{k}}$. Applying Lemma 3, we obtain

$$
\left\|x_{n_{k}}-z_{n_{k}}\right\| \leq \frac{1}{l}\left\|x_{n_{k}}-y_{n_{k}}\right\| \rightarrow 0 \text { as } k \rightarrow \infty
$$

Consequently, $z_{n_{k}} \rightarrow z \in C$, this implies that $\left\{z_{n_{k}}\right\}$ is bounded. From the uniformly continuity of the operator $A$ on bounded subsets of $H$ it follows that

$$
\left\|A x_{n_{k}}-A z_{n_{k}}\right\| \rightarrow 0 \text { as } k \rightarrow \infty .
$$

By the Armijo line-search rule (9) we must have

$$
\lambda_{n_{k}} \cdot l^{-1}\left\|A x_{n_{k}}-A P_{C}\left(x_{n_{k}}-\lambda_{n_{k}} l^{-1} A x_{n_{k}}\right)\right\|>\mu\left\|x_{n_{k}}-P_{C}\left(x_{n_{k}}-\lambda_{n_{k}} l^{-1} A x_{n_{k}}\right)\right\| .
$$

That is,

$$
\frac{1}{\mu}\left\|A x_{n_{k}}-A z_{n_{k}}\right\|>\frac{\left\|x_{n_{k}}-z_{n_{k}}\right\|}{\lambda_{n_{k}} l^{-1}} .
$$

Combining (24) and (25) we obtain

$$
\lim _{k \rightarrow \infty} \frac{\left\|x_{n_{k}}-z_{n_{k}}\right\|}{\lambda_{n_{k}} l^{-1}}=0
$$

Furthermore, we have from the definition of $z_{n_{k}}$ that

$$
\left\langle x_{n_{k}}-\lambda_{n_{k}} l^{-1} A x_{n_{k}}-z_{n_{k}}, x-z_{n_{k}}\right\rangle \leq 0 \quad \forall x \in C .
$$

This implies that

$$
\frac{1}{\lambda_{n_{k}} l^{-1}}\left\langle x_{n_{k}}-z_{n_{k}}, x-z_{n_{k}}\right\rangle+\left\langle A x_{n_{k}}, z_{n_{k}}-x_{n_{k}}\right\rangle \leq\left\langle A x_{n_{k}}, x-x_{n_{k}}\right\rangle \forall x \in C .
$$

Taking the limit $k \rightarrow \infty$ in (26) we get

$$
\liminf _{k \rightarrow \infty}\left\langle A x_{n_{k}}, x-x_{n_{k}}\right\rangle \geq 0 .
$$

Therefore, the inequality (23) is proved.

On the other hand, we have

$$
\left\langle A y_{n_{k}}, x-y_{n_{k}}\right\rangle=\left\langle A y_{n_{k}}-A x_{n_{k}}, x-x_{n_{k}}\right\rangle+\left\langle A x_{n_{k}}, x-x_{n_{k}}\right\rangle+\left\langle A y_{n_{k}}, x_{n_{k}}-y_{n_{k}}\right\rangle .
$$

From $\lim _{k \rightarrow \infty}\left\|x_{n_{k}}-y_{n_{k}}\right\|=0$ and the uniformly continuity of $A$ we get

$$
\lim _{k \rightarrow \infty}\left\|A x_{n_{k}}-A y_{n_{k}}\right\|=0
$$


which, together with (23) and (27) implies that

$$
\liminf _{k \rightarrow \infty}\left\langle A y_{n_{k}}, x-y_{n_{k}}\right\rangle \geq 0 \text {. }
$$

Next, we show that $z \in \Omega$. We choose a sequence $\left\{\varepsilon_{k}\right\}$ of positive numbers decreasing and tending to 0 . We can construct a strictly increasing sequence $\left\{N_{k}\right\}$ of positive integers such that

$$
\left\langle A y_{n_{j}}, x-y_{n_{j}}\right\rangle+\varepsilon_{k} \geq 0 \forall j \geq N_{k},
$$

where the existence of $N_{k}$ follows from (29). Furthermore, for each $k$ setting

$$
v_{N_{k}}=\frac{A y_{N_{k}}}{\left\|A y_{N_{k}}\right\|^{2}}
$$

we have $\left\langle A y_{N_{k}}, v_{N_{k}}\right\rangle=1$. We deduce from (30) that for each $k$

$$
\left\langle A y_{N_{k}}, x+\varepsilon_{k} v_{N_{k}}-y_{N_{k}}\right\rangle \geq 0 .
$$

From the fact that $A$ is pseudo-monotone on $H$, we get

$$
\left\langle A\left(x+\varepsilon_{k} v_{N_{k}}\right), x+\varepsilon_{k} v_{N_{k}}-y_{N_{k}}\right\rangle \geq 0 .
$$

This implies that

$$
\left\langle A x, x-y_{N_{k}}\right\rangle \geq\left\langle A x-A\left(x+\varepsilon_{k} v_{N_{k}}\right), x+\varepsilon_{k} v_{N_{k}}-y_{N_{k}}\right\rangle-\varepsilon_{k}\left\langle A x, v_{N_{k}}\right\rangle .
$$

We show that $\lim _{k \rightarrow \infty} \varepsilon_{k} v_{N_{k}}=0$. Indeed, since $x_{n_{k}} \rightarrow z$ and $\lim _{k \rightarrow \infty}\left\|x_{n_{k}}-y_{n_{k}}\right\|=0$, we obtain $y_{N_{k}} \rightarrow z$ as $k \rightarrow \infty$. Since $A$ is sequentially weakly continuous on bounded subset of $H,\left\{A y_{n_{k}}\right\}$ converges weakly to $A z$. We have that $A z \neq 0$ (otherwise, $z$ is a solution). Since the norm mapping is sequentially weakly lower semicontinuous, we have

$$
0<\|A z\| \leq \liminf _{k \rightarrow \infty}\left\|A y_{n_{k}}\right\| .
$$

Since $\left\{y_{N_{k}}\right\} \subset\left\{y_{n_{k}}\right\}$ and $\varepsilon_{k} \rightarrow 0$ as $k \rightarrow \infty$, we obtain

$$
0 \leq \limsup _{k \rightarrow \infty}\left\|\varepsilon_{k} v_{N_{k}}\right\|=\limsup _{k \rightarrow \infty}\left(\frac{\varepsilon_{k}}{\left\|A y_{n_{k}}\right\|}\right) \leq \frac{\limsup _{k \rightarrow \infty} \varepsilon_{k}}{\liminf _{k \rightarrow \infty}\left\|A y_{n_{k}}\right\|}=0,
$$

which implies that $\lim _{k \rightarrow \infty} \varepsilon_{k} v_{N_{k}}=0$. Letting $k \rightarrow \infty$, the right hand side of (31) tends to zero since $A$ is uniformly continuous, $\left\{x_{N_{k}}\right\}$ is bounded and $\lim _{k \rightarrow \infty} \varepsilon_{k} v_{N_{k}}=0$. Thus, we get

$$
\liminf _{k \rightarrow \infty}\left\langle A x, x-y_{N_{k}}\right\rangle \geq 0
$$

Hence, for all $x \in C$ we have

$$
\langle A x, x-z\rangle=\lim _{k \rightarrow \infty}\left\langle A x, x-y_{N_{k}}\right\rangle=\liminf _{k \rightarrow \infty}\left\langle A x, x-y_{N_{k}}\right\rangle \geq 0 .
$$

By Lemma 5 we obtain $z \in \Omega$ and the proof is complete.

Remark 2. As remarked in [3, 33], when the operator A is monotone, it is not necessary to impose the sequential weak-weak continuity on A.

Combining Lemma 9 and Lemma 10 with Lemma 6 we obtain the weak convergence of Algorithm 1.

Theorem 1. The sequence $\left\{x_{n}\right\}$ generated by Algorithm 1 converges weakly to a solution of $\operatorname{VI}(C, A)$. 


\section{Strong Convergence Method}

In this section, we introduce our second method which is a combination of Tseng's extragradient method with Mann type method [25]. Through out this section, in addition to conditions 1-3 in Section 3 we also need the following condition.

Condition 4. Let $\left\{\alpha_{n}\right\},\left\{\beta_{n}\right\}$ be two real sequences in $(0,1)$ such that $\left\{\beta_{n}\right\} \subset\left(a, 1-\alpha_{n}\right)$ for some $a>0$ and

$$
\lim _{n \rightarrow \infty} \alpha_{n}=0, \sum_{n=1}^{\infty} \alpha_{n}=\infty
$$

The proposed algorithm is of the following form:

\section{Algorithm 2.}

Initialization: Given $\gamma>0, l \in(0,1), \mu \in(0,1)$. Let $x_{1} \in C$ be arbitrary

Iterative Steps: Given the current iterate $x_{n}$, calculate $x_{n+1}$ as follows:

Step 1. Compute

$$
y_{n}=P_{C}\left(x_{n}-\lambda_{n} A x_{n}\right)
$$

where $\lambda_{n}:=\gamma l^{m_{n}}$ and $m_{n}$ is the smallest non-negative integer $m$ satisfying

$$
\gamma l^{m}\left\|A x_{n}-A y_{n}\right\| \leq \mu\left\|x_{n}-y_{n}\right\|
$$

If $x_{n}=y_{n}$ or $A y_{n}=0$ then Stop, $y_{n}$ is a solution of $\operatorname{VI}(C, A)$. Otherwise

Step 2. Compute

$$
x_{n+1}=\left(1-\alpha_{n}-\beta_{n}\right) x_{n}+\beta_{n}\left(y_{n}-\lambda_{n}\left(A y_{n}-A x_{n}\right)\right) .
$$

Set $n:=n+1$ and go to Step 1 .

Lemma 11. The sequence $\left\{x_{n}\right\}$ generated by Algorithm 2 is bounded.

Proof. Setting $z_{n}=y_{n}-\lambda_{n}\left(A y_{n}-A x_{n}\right)$. Thanks to Lemma 9, for every $p \in \Omega$ we have

$$
\left\|z_{n}-p\right\|^{2} \leq\left\|x_{n}-p\right\|^{2}-\left(1-\mu^{2}\right)\left\|x_{n}-y_{n}\right\|^{2} .
$$

This implies that

$$
\left\|z_{n}-p\right\| \leq\left\|x_{n}-p\right\|
$$

We have

$$
\begin{aligned}
\left\|x_{n+1}-p\right\| & =\left\|\left(1-\alpha_{n}-\beta_{n}\right) x_{n}+\beta_{n} z_{n}-p\right\| \\
& =\left\|\left(1-\alpha_{n}-\beta_{n}\right)\left(x_{n}-p\right)+\beta_{n}\left(z_{n}-p\right)-\alpha_{n} p\right\| \\
& \leq\left\|\left(1-\alpha_{n}-\beta_{n}\right)\left(x_{n}-p\right)+\beta_{n}\left(z_{n}-p\right)\right\|+\alpha_{n}\|p\| .
\end{aligned}
$$


From (33) we obtain

$$
\begin{aligned}
\|( & \left.-\alpha_{n}-\beta_{n}\right)\left(x_{n}-p\right)+\beta_{n}\left(z_{n}-p\right) \|^{2} \\
& =\left(1-\alpha_{n}-\beta_{n}\right)^{2}\left\|x_{n}-p\right\|^{2}+2\left(1-\alpha_{n}-\beta_{n}\right) \beta_{n}\left\langle x_{n}-p, z_{n}-p\right\rangle+\beta_{n}^{2}\left\|z_{n}-p\right\|^{2} \\
& \leq\left(1-\alpha_{n}-\beta_{n}\right)^{2}\left\|x_{n}-p\right\|^{2}+2\left(1-\alpha_{n}-\beta_{n}\right) \beta_{n}\left\|z_{n}-p\right\|\left\|x_{n}-p\right\|+\beta_{n}^{2}\left\|z_{n}-p\right\|^{2} \\
& \leq\left(1-\alpha_{n}-\beta_{n}\right)^{2}\left\|x_{n}-p\right\|^{2}+2\left(1-\alpha_{n}-\beta_{n}\right) \beta_{n}\left\|x_{n}-p\right\|^{2}+\beta_{n}^{2}\left\|x_{n}-p\right\|^{2} \\
& =\left(1-\alpha_{n}\right)^{2}\left\|x_{n}-p\right\|^{2} .
\end{aligned}
$$

This implies that

$$
\left\|\left(1-\alpha_{n}-\beta_{n}\right)\left(x_{n}-p\right)+\beta_{n}\left(z_{n}-p\right)\right\| \leq\left(1-\alpha_{n}\right)\left\|x_{n}-p\right\| .
$$

Combining (34) and (35) we get

$$
\begin{aligned}
\left\|x_{n+1}-p\right\| & \leq\left(1-\alpha_{n}\right)\left\|x_{n}-p\right\|+\alpha_{n}\|p\| \\
& \leq \max \left\{\left\|x_{n}-p\right\|,\|p\|\right\} \\
& \leq \ldots \leq \max \left\{\left\|x_{0}-p\right\|,\|p\|\right\} .
\end{aligned}
$$

i.e., the sequence $\left\{x_{n}\right\}$ is bounded and so is $\left\{z_{n}\right\}$.

Lemma 12. For every $p \in \Omega$ we have

$$
\left\|x_{n+1}-p\right\|^{2} \leq\left(1-\alpha_{n}\right)\left\|x_{n}-p\right\|^{2}+\alpha_{n}\left[2 \beta_{n}\left\|x_{n}-z_{n}\right\|\left\|x_{n+1}-p\right\|+2\left\langle p, p-x_{n+1}\right\rangle\right] .
$$

Proof. Using (2) we have

$$
\begin{aligned}
\left\|x_{n+1}-p\right\|^{2}= & \left\|\left(1-\alpha_{n}-\beta_{n}\right) x_{n}+\beta_{n} z_{n}-p\right\|^{2} \\
= & \left\|\left(1-\alpha_{n}-\beta_{n}\right)\left(x_{n}-p\right)+\beta_{n}\left(z_{n}-p\right)+\alpha_{n}(-p)\right\|^{2} \\
= & \left(1-\alpha_{n}-\beta_{n}\right)\left\|x_{n}-p\right\|^{2}+\beta_{n}\left\|z_{n}-p\right\|^{2}+\alpha_{n}\|p\|^{2}-\beta_{n}\left(1-\alpha_{n}-\beta_{n}\right)\left\|x_{n}-z_{n}\right\|^{2} \\
& -\alpha_{n}\left(1-\alpha_{n}-\beta_{n}\right)\left\|x_{n}\right\|^{2}-\alpha_{n} \beta_{n}\left\|z_{n}\right\|^{2} \\
\leq & \left(1-\alpha_{n}-\beta_{n}\right)\left\|x_{n}-p\right\|^{2}+\beta_{n}\left\|z_{n}-p\right\|^{2}+\alpha_{n}\|p\|^{2}
\end{aligned}
$$

which, together (32) implies

$$
\begin{aligned}
\left\|x_{n+1}-p\right\|^{2} & \leq\left(1-\alpha_{n}-\beta_{n}\right)\left\|x_{n}-p\right\|^{2}+\beta_{n}\left\|x_{n}-p\right\|^{2}-\beta_{n}\left(1-\mu^{2}\right)\left\|x_{n}-y_{n}\right\|^{2}+\alpha_{n}\|p\|^{2} \\
& =\left(1-\alpha_{n}\right)\left\|x_{n}-p\right\|^{2}-\beta_{n}\left(1-\mu^{2}\right)\left\|x_{n}-y_{n}\right\|^{2}+\alpha_{n}\|p\|^{2} \\
& \leq\left\|x_{n}-p\right\|^{2}-\beta_{n}\left(1-\mu^{2}\right)\left\|x_{n}-y_{n}\right\|^{2}+\alpha_{n}\|p\|^{2} .
\end{aligned}
$$

Therefore,

$$
\beta_{n}\left(1-\mu^{2}\right)\left\|x_{n}-y_{n}\right\|^{2} \leq\left\|x_{n}-p\right\|^{2}-\left\|x_{n+1}-p\right\|^{2}+\alpha_{n}\|p\|^{2} .
$$

Setting $t_{n}=\left(1-\beta_{n}\right) x_{n}+\beta_{n} z_{n}$ we obtain

$$
\begin{aligned}
\left\|t_{n}-p\right\| & =\left\|\left(1-\beta_{n}\right)\left(x_{n}-p\right)+\beta_{n}\left(z_{n}-p\right)\right\| \\
& \leq\left(1-\beta_{n}\right)\left\|x_{n}-p\right\|+\beta_{n}\left\|z_{n}-p\right\| \\
& \leq\left(1-\beta_{n}\right)\left\|x_{n}-p\right\|+\beta_{n}\left\|x_{n}-p\right\| \\
& =\left\|x_{n}-p\right\|,
\end{aligned}
$$


and

$$
\left\|t_{n}-x_{n}\right\|=\beta_{n}\left\|x_{n}-z_{n}\right\| .
$$

Combining (40) and (41) we get

$$
\begin{aligned}
\left\|x_{n+1}-p\right\|^{2} & =\left\|\left(1-\alpha_{n}-\beta_{n}\right) x_{n}+\beta_{n} z_{n}-p\right\|^{2} \\
& =\left\|\left(1-\beta_{n}\right) x_{n}+\beta_{n} z_{n}-\alpha_{n} x_{n}-p\right\|^{2} \\
& =\left\|\left(1-\alpha_{n}\right)\left(t_{n}-p\right)-\alpha_{n}\left(x_{n}-t_{n}\right)-\alpha_{n} p\right\|^{2} \\
& \leq\left(1-\alpha_{n}\right)^{2}\left\|t_{n}-p\right\|^{2}-2\left\langle\alpha_{n}\left(x_{n}-t_{n}\right)+\alpha_{n} p, x_{n+1}-p\right\rangle \\
& =\left(1-\alpha_{n}\right)^{2}\left\|t_{n}-p\right\|^{2}+2 \alpha_{n}\left\langle x_{n}-t_{n}, p-x_{n+1}\right\rangle+2 \alpha_{n}\left\langle p, p-x_{n+1}\right\rangle \\
& \leq\left(1-\alpha_{n}\right)\left\|t_{n}-p\right\|^{2}+2 \alpha_{n}\left\|x_{n}-t_{n}\right\|\left\|x_{n+1}-p\right\|+2 \alpha_{n}\left\langle p, p-x_{n+1}\right\rangle \\
& \leq\left(1-\alpha_{n}\right)\left\|x_{n}-p\right\|^{2}+\alpha_{n}\left[2 \beta_{n}\left\|x_{n}-z_{n}\right\|\left\|x_{n+1}-p\right\|+2\left\langle p, p-x_{n+1}\right\rangle\right] .
\end{aligned}
$$

We are now in the position to establish the main results of this section. To this end, we assume that Algorithm 2 does not terminate at any step $n$, i.e., it generates an infinite sequence $\left\{x_{n}\right\}$.

Theorem 2. Suppose that Algorithm 2 generates an infinite iterative sequence $\left\{x_{n}\right\}$ then $\left\{x_{n}\right\}$ converges strongly to $p \in \Omega$, where $p=\arg \min \{\|z\|: z \in \Omega\}$.

Proof. Since $\Omega$ is closed and convex [18], there exists an unique element $p \in \Omega$ such that $p=P_{\Omega}(0)$. We will show that the sequence $\left\{\left\|x_{n}-p\right\|^{2}\right\}$ converges to zero by considering two possible cases on the sequence $\left\{\left\|x_{n}-p\right\|^{2}\right\}$.

Case 1: There exists an $n_{0} \in \mathbb{N}$ such that $\left\|x_{n+1}-p\right\|^{2} \leq\left\|x_{n}-p\right\|^{2}$ for all $n \geq n_{0}$. This implies that $\lim _{n \rightarrow \infty}\left\|x_{n}-p\right\|^{2}$ exists. It implies from Claim 2 that

$$
\lim _{n \rightarrow \infty}\left\|x_{n}-y_{n}\right\|=0 \text {. }
$$

We also have

$$
\begin{aligned}
\left\|z_{n}-x_{n}\right\| & =\left\|y_{n}-\lambda_{n}\left(A y_{n}-A x_{n}\right)-x_{n}\right\| \\
& \leq(1+\mu)\left\|x_{n}-y_{n}\right\| .
\end{aligned}
$$

Combining (42) and (43) we get

$$
\lim _{n \rightarrow \infty}\left\|z_{n}-x_{n}\right\|=0
$$

Using this we find

$$
\left\|x_{n+1}-x_{n}\right\| \leq \alpha_{n}\left\|x_{n}\right\|+\beta_{n}\left\|x_{n}-z_{n}\right\| \rightarrow 0 \text { as } n \rightarrow \infty .
$$

Since $\left\{x_{n}\right\}$ is bounded we assume that there exists a subsequence $\left\{x_{n_{j}}\right\}$ of $\left\{x_{n}\right\}$ such that $x_{n_{j}} \rightarrow q$ and

$$
\limsup _{n \rightarrow \infty}\left\langle p, p-x_{n}\right\rangle=\lim _{j \rightarrow \infty}\left\langle p, p-x_{n_{j}}\right\rangle=\langle p, p-q\rangle .
$$

We have $x_{n_{j}} \rightarrow q$ and $\left\|x_{n}-y_{n}\right\| \rightarrow 0$, using the same technique as in Lemma 10 we get $q \in \Omega$. On the other hand, since $p=P_{\Omega} 0$, we obtain

$$
\limsup _{n \rightarrow \infty}\left\langle p, p-x_{n}\right\rangle=\langle p, p-q\rangle \leq 0 .
$$


By $\left\|x_{n+1}-x_{n}\right\| \rightarrow 0$ we get

$$
\limsup _{n \rightarrow \infty}\left\langle p, p-x_{n+1}\right\rangle \leq 0 .
$$

From Lemma 12 and Lemma 7 we have $\lim _{n \rightarrow \infty}\left\|x_{n}-p\right\|^{2}=0$, i.e., $x_{n} \rightarrow p$.

Case 2: Assume that there is no $n_{0} \in \mathbb{N}$ such that $\left\{\left\|x_{n}-p\right\|\right\}_{n=n_{0}}^{\infty}$ is monotonically decreasing. Following the technique in [22] we define $\Gamma_{n}=\left\|x_{n}-p\right\|^{2}$ for all $n \geq 1$ and let $\tau: \mathbb{N} \rightarrow \mathbb{N}$ be a mapping defined for all $n \geq n_{0}$ (for some $n_{0}$ large enough) by

$$
\tau(n):=\max \left\{k \in \mathbb{N}: k \leq n, \Gamma_{k} \leq \Gamma_{k+1}\right\},
$$

i.e. $\tau(n)$ is the largest number $k$ in $\{1, \ldots, n\}$ such that $\Gamma_{k}$ increases at $k=\tau(n)$; note that, in view of Case 2, this $\tau(n)$ is well-defined for all sufficiently large $n$. From [22], $\tau$ is a non-decreasing sequence such that $\tau(n) \rightarrow \infty$ as $n \rightarrow \infty$ and for all $n \geq n_{0}$

$$
\begin{gathered}
0 \leq \Gamma_{\tau(n)} \leq \Gamma_{\tau(n)+1}, \\
0 \leq \Gamma_{n} \leq \Gamma_{\tau(n)+1} .
\end{gathered}
$$

Since $\beta_{n} \geq a \forall n \in \mathbb{N}$, from (39) we have

$$
\begin{aligned}
a\left(1-\mu^{2}\right)\left\|x_{\tau(n)}-y_{\tau(n)}\right\|^{2} & \leq \beta_{\tau(n)}\left(1-\mu^{2}\right)\left\|x_{\tau(n)}-y_{\tau(n)}\right\|^{2} \\
& \leq\left\|x_{\tau(n)}-p\right\|^{2}-\left\|x_{\tau(n)+1}-p\right\|^{2}+\alpha_{\tau(n)}\|p\|^{2} \\
& \leq \alpha_{\tau(n)}\|p\|^{2} .
\end{aligned}
$$

Therefore

$$
\lim _{n \rightarrow \infty}\left\|x_{\tau(n)}-y_{\tau(n)}\right\|=0
$$

As proved in the first case, we have

$$
\left\|x_{\tau(n)+1}-x_{\tau(n)}\right\| \rightarrow 0
$$

and

$$
\limsup _{n \rightarrow \infty}\left\langle p, p-x_{\tau(n)+1}\right\rangle \leq 0 .
$$

From Lemma 12 and $\Gamma_{\tau(n)} \leq \Gamma_{\tau(n)+1} \forall n \geq n_{0}$ we have

$$
\begin{aligned}
\left\|x_{\tau(n)+1}-p\right\|^{2} \leq & \left(1-\alpha_{\tau(n)}\right)\left\|x_{\tau(n)}-p\right\|^{2} \\
& +\alpha_{\tau(n)}\left[2 \beta_{\tau(n)}\left\|x_{\tau(n)}-z_{\tau(n)}\right\|\left\|x_{\tau(n)+1}-p\right\|+2\left\langle p, p-x_{\tau(n)+1}\right\rangle\right] \\
\leq & \left(1-\alpha_{\tau(n)}\right)\left\|x_{\tau(n)+1}-p\right\|^{2} \\
& +\alpha_{\tau(n)}\left[2 \beta_{\tau(n)}\left\|x_{\tau(n)}-z_{\tau(n)}\right\|\left\|x_{\tau(n)+1}-p\right\|+2\left\langle p, p-x_{\tau(n)+1}\right\rangle\right] .
\end{aligned}
$$

This implies that

$$
\left\|x_{\tau(n)+1}-p\right\|^{2} \leq 2 \beta_{\tau(n)}\left\|x_{\tau(n)}-z_{\tau(n)}\right\|\left\|x_{\tau(n)+1}-p\right\|+2\left\langle p, p-x_{\tau(n)+1}\right\rangle,
$$

which implies that $\limsup _{n \rightarrow \infty}\left\|x_{\tau(n)+1}-p\right\|^{2} \leq 0$, that is $\lim _{n \rightarrow \infty}\left\|x_{\tau(n)+1}-p\right\|=0$. The conclusion is follows from (44).

Remark 3. Comparing with Theorem 3.1 in [37], Theorem 3.2 in [29] and Theorem 3.2 in [35], Theorem 2 has two major advantages.

1. We weaken the Lipschitz continuity of A to uniform continuity on bounded subsets.

2. We replace the monotonicity by pseudo-monotonicity and sequentially weakly continuous of $A$. 


\section{Numerical Illustrations}

In this section we present some numerical examples illustrating the behavior of our proposed schemes. We consider the classical Hilbert space $H=l_{2}$ and the $\operatorname{VI}(C, A)$ with

$$
C:=\left\{x=\left(x_{1}, x_{2}, \ldots, x_{i}, \ldots\right) \in H|| x_{i} \mid \leq \frac{1}{i}, i=1,2, \ldots, n, \ldots\right\}
$$

and

$$
A x:=\left(\|x\|+\frac{1}{\|x\|+\alpha}\right) x,
$$

for some $\alpha>0$. It is easy to see that $\Omega=\{0\}$ and moreover, $A$ is pseudo-monotone on $H$, uniformly continuous and sequentially weakly continuous on $C$ but not Lipschitz continuous on $H$. Indeed, let $x, y \in H$ be such that $\langle A x, y-x\rangle \geq 0$. This implies that $\langle x, y-x\rangle \geq 0$. Consequently,

$$
\begin{aligned}
\langle A y, y-x\rangle & =\left(\|x\|+\frac{1}{\|x\|+\alpha}\right)\langle y, y-x\rangle \\
& \geq\left(\|x\|+\frac{1}{\|x\|+\alpha}\right)(\langle y, y-x\rangle-\langle x, y-x\rangle) \\
& =\left(\|x\|+\frac{1}{\|x\|+\alpha}\right)\|y-x\|^{2} \geq 0 .
\end{aligned}
$$

meaning that $A$ is pseudo-monotone. Moreover, since $C$ is compact, the operator $A$ is uniformly continuous and sequentially weakly continuous on $C$. Finally we show that $A$ is not Lipschitz continuous on $H$. Assume to the contrary that $A$ is Lipschitz continuous on $H$, i.e., there exists $L>0$ such that

$$
\|A x-A y\| \leq L\|x-y\| \forall x, y \in H .
$$

Let $x=(L, 0, \ldots, 0, \ldots)$ and $y=(0,0, \ldots, 0, \ldots)$ then

$$
\|A x-A y\|=\|A x\|=\left(\|x\|+\frac{1}{\|x\|+\alpha}\right)\|x\|=\left(L+\frac{1}{L+\alpha}\right) L .
$$

Thus, $\|A x-A y \mid \leq L\| x-y \|$ is equivalent to

$$
\left(L+\frac{1}{L+\alpha}\right) L \leq L^{2}
$$

leading to

$$
\frac{1}{L+\alpha} \leq 0,
$$

which is a contraction and thus $A$ is not Lipschitz continuous on $H$. In the following figure, we present the numerical behavior of Algorithm 1 and Algorithm 2 when $\alpha=1$, $H=\mathbb{R}^{m}$ for different values of $m$. In this case, the feasible set $C$ is a box

$$
C:=\left\{x \in \mathbb{R}^{m} \mid \frac{-1}{i} \leq x_{i} \leq \frac{1}{i}, i=1,2, \ldots, m\right\},
$$


for which we have explicit formula of the projection onto $C$. We choose $\gamma=0.1, l=$ $0.5, \mu=0.8$ for both Algorithms and

$$
\alpha_{n}=\frac{1}{\sqrt{n}+2}, \beta_{n}=\frac{1-\alpha_{n}}{2} \quad \forall n \in \mathbb{N},
$$

for Algorithm 2. This choice of parameters implies that (9) is satisfied with $m_{n}=0$ for all iterations. The starting point is chosen as $x_{0}=(1,1, \ldots 1) \in \mathbb{R}^{m}$. Experiments were generated with Matlab R2017a on a Linux OS with a $2.39 \mathrm{Ghz}$ processor and $16 \mathrm{~GB}$ of memory. When $m=20$, Algorithm 1 provides the solution after 149 iterations and 0.001746 seconds of CPU time, while Algorithm 2 needs 71 iterations and 0.001022 seconds of CPU time. When $m=50$, Algorithm 1 provides the solution after 150 iterations and 0.001784 seconds of CPU time, while Algorithm 2 needs 73 iterations and 0.001191 seconds of CPU time.
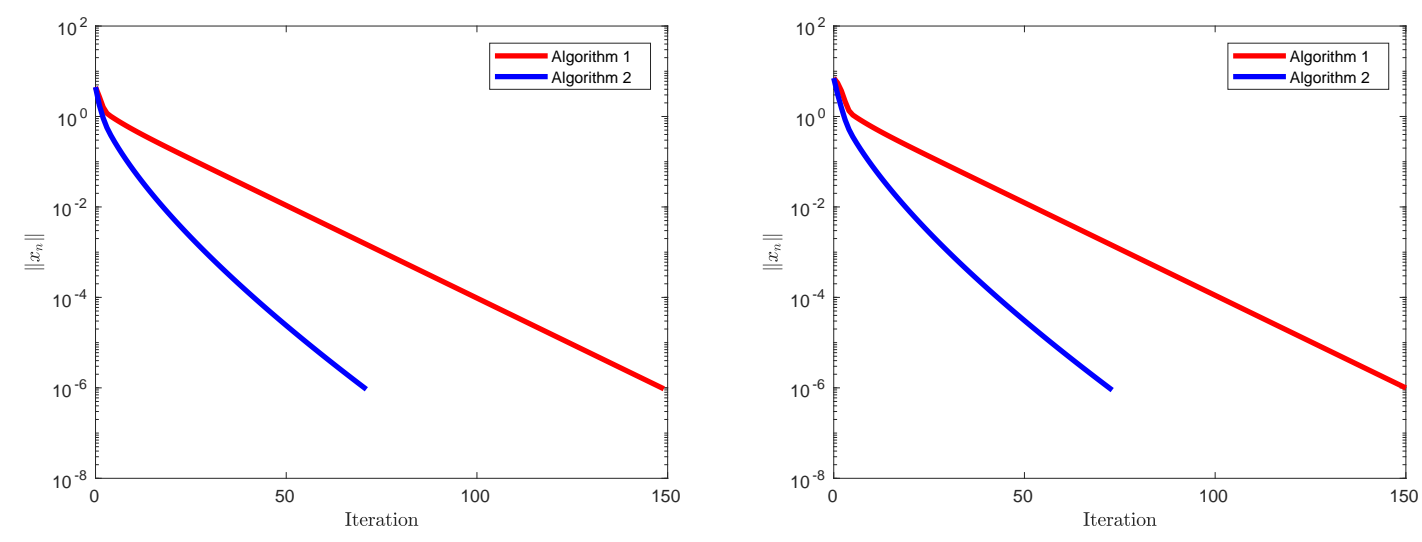

Figure 1: Performance of Algorithm 1 and Algorithm 2 when $m=20$ (left) and $m=50$ (right).

\section{Conclusions}

In this paper we proposed two Tseng's extragradient extensions for solving non-Lipschitzian pseudo-monotone variational inequalities in real Hilbert spaces. Under suitable and standard conditions we establish weak and strong convergence theorems of the proposed schemes. Our results extend and generalize some existing results in the literature and numerical illustrations demonstrate the behavior and potential applicability of the proposed methods.

\section{Acknowledgment}

We thank the anonymous reviewers for their constructive comments which help us improve the presentation of this paper. We would also like to thank the Associate Editor for the efficient handling of the review process. This work was supported by the Vietnam National Foundation for Science and Technology Development (NAFOSTED) grant 101.01-2017.315 and the Austrian Science Foundation (FWF), grant M-2499. 


\section{References}

[1] Antipin, A. S.: On a method for convex programs using a symmetrical modification of the Lagrange function. Ekonomika i Mat. Metody. 12, 1164-1173 (1976)

[2] Bauschke, H. H., Combettes, P. L.: Convex Analysis and Monotone Operator Theory in Hilbert Spaces. CMS Books in Mathematics, Springer, New York (2011)

[3] Boţ, R. I., Csetnek, E. R., Vuong, P. T.: The Forward-Backward-Forward Method from discrete and continuous perspective for pseudo-monotone variational inequalities in Hilbert Spaces. arXiv:1808.08084 (2018)

[4] Censor, Y., Gibali A, Reich S.: The subgradient extragradientmethod for solving variational inequalities in Hilbert space. J. Optim. Theory Appl. 148, 318-335 (2011)

[5] Censor, Y., Gibali, A., Reich, S.: Strong convergence of subgradient extragradient methods for the variational inequality problem in Hilbert space. Optim. Meth. Softw. 26, 827-845 (2011)

[6] Censor, Y., Gibali, A., Reich, S.: Extensions of Korpelevich's extragradient method for the variational inequality problem in Euclidean space. Optimization. 61, 1119$1132(2011)$

[7] Ceng, L. C., Teboulle, M., Yao, Y.: Weak convergence of an iterative method for pseudomonotone variational inequalities and fixed-point problems. J. Optim. Theory Appl. 146, 19-31 (2010)

[8] Cottle, R.W., Yao, J.C.: Pseudo-monotone complementarity problems in Hilbert space. J. Optim. Theory Appl. 75, 281-295 (1992)

[9] Denisov, S.V., Semenov, V.V., Chabak, L.M.: Convergence of the modified extragradient method for variational inequalities with non-Lipschitz operators. Cybern. Syst. Anal. 51, 757-765 (2015)

[10] Facchinei, F., Pang, J.S.: Finite-Dimensional Variational Inequalities and Complementarity Problems. Springer Series in Operations Research, vols. I and II. Springer, New York (2003)

[11] Fichera, G.: Sul problema elastostatico di Signorini con ambigue condizioni al contorno. Atti Accad. Naz. Lincei, VIII. Ser., Rend., Cl. Sci. Fis. Mat. Nat. 34, 138-142 (1963)

[12] Fichera, G.: Problemi elastostatici con vincoli unilaterali: il problema di Signorini con ambigue condizioni al contorno. Atti Accad. Naz. Lincei, Mem., Cl. Sci. Fis. Mat. Nat., Sez. I, VIII. Ser. 7, 91-140 (1964)

[13] Goebel, K., Reich, S.: Uniform Convexity, Hyperbolic Geometry, and Nonexpansive Mappings. Marcel Dekker, New York 1984 
[14] Iusem, A.N., Gárciga Otero, R.: Inexact versions of proximal point and augmented Lagrangian algorithms in Banach spaces. Numer. Funct. Anal. Optim., 22, 609-640 (2001)

[15] Iusem, A.N., Nasri, M.: Korpelevich's method for variational inequality problems in Banach spaces. J. Glob. Optim., 50, 59-76 (2011)

[16] Khanh, P.D., Vuong, P. T.: Modified projection method for strongly pseudomonotone variational inequalities. J. Global Optim. 58, 341-350 (2014)

[17] Kim, D. S., Khanh, P.D., Vuong, P. T.: Qualitative properties of strongly pseudomonotone variational inequalities. Opt. Lett. 10, 341-350 (2016)

[18] Kinderlehrer, D., Stampacchia, G.: An Introduction to Variational Inequalities and Their Applications. Academic Press, New York, (1980)

[19] Konnov, I. V.: Combined Relaxation Methods for Variational Inequalities. SpringerVerlag, Berlin, (2001).

[20] Korpelevich, G.M.: The extragradient method for finding saddle points and other problems. Ekonomika i Mat. Metody. 12, 747-756 (1976)

[21] Liu, L.S.: Ishikawa and Mann iteration process with errors for nonlinear strongly accretive mappings in Banach space. J. Math. Anal. Appl. 194, 114-125 (1995)

[22] Maingé, P.E.: A hybrid extragradient-viscosity method for monotone operators and fixed point problems. SIAM J. Control Optim. 47, 1499-1515 (2008)

[23] Malitsky, Y.V.: Projected reflected gradient methods for monotone variational inequalities. SIAM J. Optim. 25, 502-520 (2015)

[24] Malitsky, Y.V., Semenov, V.V.: A hybrid method without extrapolation step for solving variational inequality problems. J. Glob. Optim. 61, 193-202 (2015)

[25] Mann, W.R.: Mean value methods in iteration. Proc. Amer. Math. Soc. 4, 506-510 (1953)

[26] Opial, Z.: Weak convergence of the sequence of successive approximations for nonexpansive mappings. Bulletin of the American Mathematical Society, 73, 591-597 (1967)

[27] Reich, S.: Constructive techniques for accretive and monotone operators. Applied Nonlinear Analysis. Academic Press, New York, 335-345 (1979)

[28] Solodov, M.V., Svaiter, B.F.: A new projection method for variational inequality problems. SIAM J. Control Optim. 37, 765-776 (1999)

[29] Thong, D.V., Hieu, D.V.: Weak and strong convergence theorems for variational inequality problems. Numer. Algorithms. 78, 1045-1060 (2018)

[30] Thong, D.V., Hieu, D.V.: Modified subgradient extragradient method for inequality variational problems. Numer. Algorithms. 79, 597-610 (2018) 
[31] Thong, D.V., Hieu, D.V.: Inertial extragradient algorithms for strongly pseudomonotone variational inequalities. J. Comput. Appl. Math. 341, 80-98 (2018)

[32] Tseng, P.: A modified forward-backward splitting method for maximal monotone mappings. SIAM J. Control Optim. 38, 431-446 (2000)

[33] Vuong, P.T.: On the weak convergence of the extragradient method for solving pseudo-monotone variational inequalities. J. Optim. Theory Appl. 176, 399-409 (2018)

[34] Vuong, P.T., Shehu, Y.: Convergence of an extragradient-type method for variational inequality with applications to optimal control problems. Numer. Algorithms. DOI:10.1007/s11075-018-0547-6 (2018)

[35] Wang, F.H., Xu, H.K.: Weak and strong convergence theorems for variational inequality and fixed point problems with Tseng's extragradient method. Taiwanese J. Math. 16, 1125-1136 (2012)

[36] Xu, H.K.: Iterative algorithms for nonlinear operators. J. Lond. Math. Soc. 66, 240$256(2002)$

[37] Yang, J., Liu, H.: Strong convergence result for solving monotone variational inequalities in Hilbert space, Numer. Algorithms. DOI:10.1007/s11075-018-0504-4 (2018) 